\title{
A SUBSTELLAR COMMON PROPER-MOTION COMPANION TO THE PLEIAD H II 1348
}

\author{
Kerstin Geißler ${ }^{1}$, Stanimir A. Metchev ${ }^{1}$, Alfonse Pham ${ }^{2}$, James E. Larkin ${ }^{3}$, \\ Michael McElwain ${ }^{4}$, and Lynne A. Hillenbrand ${ }^{5}$ \\ ${ }^{1}$ Department of Physics and Astronomy, Stony Brook University, Stony Brook, NY 11794-3800, USA; geissler@mail.astro.sunysb.edu \\ ${ }^{2}$ Center for Exploration of Energy and Matter, Indiana University, Bloomington, IN 47408-1398, USA \\ ${ }^{3}$ Department of Physics and Astronomy, University of California, Los Angeles, CA 90095-1562, USA \\ ${ }^{4}$ Astrophysics Science Division, NASA Goddard Space Flight Center, Laboratory for Exoplanets and Stellar Astrophysics, Greenbelt, MD 20771, USA \\ ${ }^{5}$ Department of Physics, Mathematics, and Astronomy, MC 105-24, California Institute of Technology, Pasadena, CA 91125, USA \\ Received 2011 October 13; accepted 2011 December 7; published 2012 January 23
}

\begin{abstract}
We announce the identification of a proper-motion companion to the star H II 1348, a K5 V member of the Pleiades open cluster. The existence of a faint point source 1".1 away from H II 1348 was previously known from adaptive optics imaging by Bouvier et al. However, because of a high likelihood of background star contamination and in the absence of follow-up astrometry, Bouvier et al. tentatively concluded that the candidate companion was not physically associated with $\mathrm{H}$ II 1348 . We establish the proper-motion association of the pair from adaptive optics imaging with the Palomar $5 \mathrm{~m}$ telescope. Adaptive optics spectroscopy with the integral field spectrograph OSIRIS on the Keck $10 \mathrm{~m}$ telescope reveals that the companion has a spectral type of M8 \pm 1 . According to substellar evolution models, the M8 spectral type resides within the substellar mass regime at the age of the Pleiades. The primary itself is a known double-lined spectroscopic binary, which makes the resolved companion, H II 1348B, the least massive and widest component of this hierarchical triple system and the first substellar companion to a stellar primary in the Pleiades.
\end{abstract}

Key words: binaries: visual - brown dwarfs - instrumentation: adaptive optics - stars: individual $(\mathrm{Cl}$ Melotte 22 1348) - stars: low-mass

\section{INTRODUCTION}

As one of the nearest young (125 Myr; Stauffer et al. 1998) open clusters, the Pleiades have long been recognized as an important astrophysical laboratory for studying stellar evolution and the dynamics of stellar associations. Multiplicity studies of the Pleiades have focused both on stellar (e.g., Stauffer 1984; Mermilliod et al. 1992; Bouvier et al. 1997) and substellar (e.g., Martín et al. 2000; Bouy et al. 2006) members of the cluster. However, mixed star-brown dwarf (BD) systems have not been identified. One of the most extensive studies is the adaptive optics imaging survey of Bouvier et al. (1997). Conducted in the near-IR, it covered $144 \mathrm{G}$ and $\mathrm{K}$ stars to a relatively shallow depth. As a result, its sensitivity encompassed only stellar and massive substellar companions. A systematic highcontrast imaging survey of the Pleiades on a similar scale but at a higher sensitivity has not been performed since. Consequently, no substellar companions are known to $>0.2 M_{\odot}$ stars in the Pleiades. With the frequency of wide substellar companions to field-aged Sun-like stars now estimated at $\approx 3 \%\left(0.012-0.072 M_{\odot}\right.$ brown dwarfs in $28-1590$ AU orbits; Metchev \& Hillenbrand 2009), the frequency of brown dwarf secondaries around Sun-like stars in the Pleiades is expected to be comparable.

In the present paper, we announce the identification of a low-mass companion to the Pleiad H II 1348, a K5 V double-lined spectroscopic binary (Queloz et al. 1998, hereafter referred to as the primary or $\mathrm{H}$ II $1348 \mathrm{~A}$ ). The faint companion, H II 1348B, was already detected by Bouvier et al. (1997). However, without follow-up astrometric observations and due to a non-negligible probability of background star contamination, Bouvier et al. (1997) conservatively assumed that the candidate companion was an unrelated background star. The astrometric measurements confirm the proper-motion association of the pair, and AO spectra obtained at Palomar and Keck reveal that the companion has a spectral type of M8.

\section{OBSERVATIONS AND DATA REDUCTION}

\subsection{Astrometry and Photometry}

H II 1348 ( $V=12.6 \mathrm{mag})$ was targeted in conjunction with a large-scale natural-guide star AO imaging survey of young Sun-like stars, conducted with the PHARO camera (Hayward et al. 2001) on the Palomar Hale telescope. The data acquisition and reduction followed standard near-IR imaging practices and are described in Metchev \& Hillenbrand (2004) and Metchev (2006). Diffraction-limited $J, H$, and $K_{s}$ AO imaging of $\mathrm{H}$ II 1348 was taken at Palomar on 2004 October 3, in which the tertiary companion is visible $\sim 1$ '. 1 to the north (Figure 1). To determine the astrometric association of the system we used a precise calibration of the pixel scale of the PHARO camera (Metchev 2006; Metchev \& Hillenbrand 2009), obtained over time from observations of suggested astrometric calibration systems from the Sixth Catalog of Orbits of Visual Binary Stars (Hartkopf \& Mason 2011).

H II 1348 was also targeted as part of a demonstration project for the then newly commissioned OSIRIS integral field spectrograph (Larkin et al. 2006). We obtained 1.18-1.35 $\mu \mathrm{m}$ $(\mathrm{Jbb})$ and $1.47-1.80 \mu \mathrm{m}(\mathrm{Hbb})$ diffraction-limited integral field spectra of the pair with the Keck AO system (Wizinowich et al. 2000 ) on 2005 November 21 . We used the 35 mas lenslet scale, which allowed a 0 '.56 $\times 2$ '.24 field of view (FOV) with the custom broad band $J(J b b)$ and $H(H b b)$ filters in OSIRIS. The spectroscopic reduction of the integral field data cube is detailed in Section 2.3. Here, we only describe the use of the OSIRIS $H b b$-band data for astrometry.

The 35 mas scale of OSIRIS significantly undersamples the 35 mas width of the diffraction-limited Keck AO point spread 


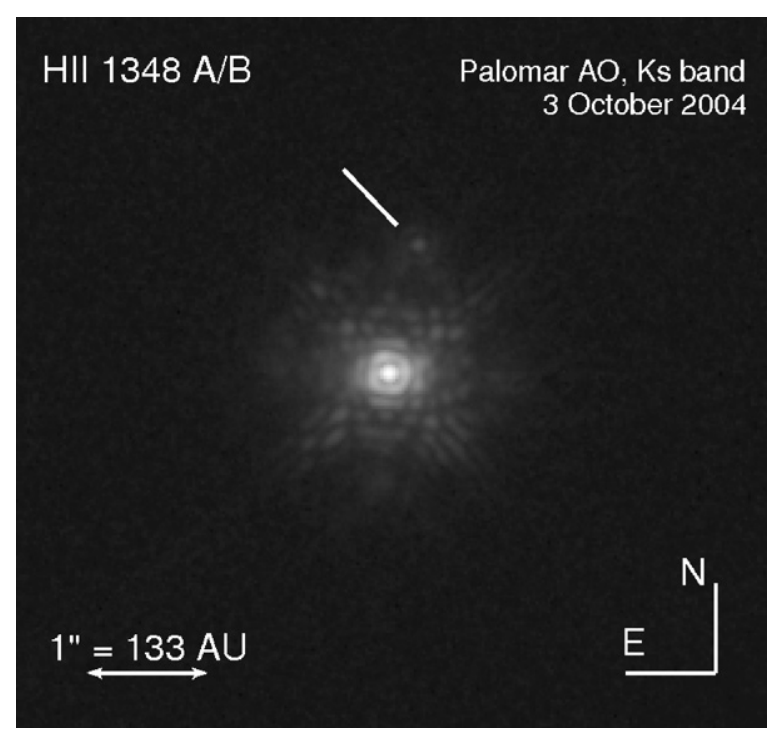

Figure 1. $K_{s}$-band image of $\mathrm{H}$ II $1348 \mathrm{~A} / \mathrm{B}$ taken with the $\mathrm{AO}$ system on the Palomar $5 \mathrm{~m}$ telescope. The Strehl ratio is $\approx 40 \%$, and the FWHM of the PSF is $0 !$ ' 10 . The companion is indicated with the white arrow. The total exposure time is $21 \mathrm{~s}$, taken as five $4.2 \mathrm{~s}$ exposures.

function (PSF) in the $H b b$ filter (Figure 2), and hence subpixel precision astrometry requires sub-pixel dithers. We did not perform such dithers during data acquisition. However, during the data analysis we noted that the spatial positions of the binary components varied monotonically between the short- and the long-wavelength end of the $H b b$ data cube, the difference spanning six lenslets $\left(0{ }^{\prime} 2\right)$ across the 1651 wavelength channels. Since the observations were conducted at an airmass of $\sim 1.05$ differential atmospheric refraction is negligible and the effect is caused by differential refraction arising from the wave front sensor dichroic in the Keck AO system. (The dichroic is oriented at $\approx 45^{\circ}$ with respect to the telescope optical axis, and hence the transmitted science light is refracted in a wavelength-dependent fashion.) The benefit to us was that the resulting spatial shift very gradually and finely sampled the lenslet size, and hence easily allowed sub-pixel precision astrometric measurements. The lenslet scale and orientation of the OSIRIS integral field spectrograph have not been calibrated on sky. We estimated systematic uncertainties of $1 \%$ in the lenslet scale and 0.3 in the detector orientation. These increased the overall positional errors determined from the OSIRIS data by a factor of $\sim 50 \%$.

Relative photometry and astrometry for the H II 1348A/B pair are given in Table 1 . The angular offset and separation between the two components have not changed significantly since the discovery of the candidate companion in 1996 (Bouvier et al. 1997), whereas a much more significant change would have been expected if the faint candidate companion were an unrelated background star (Figure 3).

\subsection{Spectroscopy: Long Slit}

We obtained $K$-band long-slit spectra of H II 1348A and B with the Palomar AO system (Dekany et al. 1998; Troy et al. 2000) and PHARO (Hayward et al. 2001) on 2004 October 3. The system was aligned along the $0{ }^{\prime} \cdot 13$ wide slit and was nodded once along an ABC-CBA pattern, for an exposure totaling 60 minutes. After pairwise subtraction, a first-order polynomial was fit to the trace of the primary, which was used to extract the spectra of both visual components. The extraction apertures were $0^{\prime \prime} 40$ and $0^{\prime \prime} 12$ wide for the primary and secondary, respectively. The FWHM of the PSF was $00^{\prime} 10$. Since H II 1348B lies in the halo of the $\sim 5$ mag brighter $\mathrm{H}$ II 1348A, we estimated and subtracted the flux from the halo of

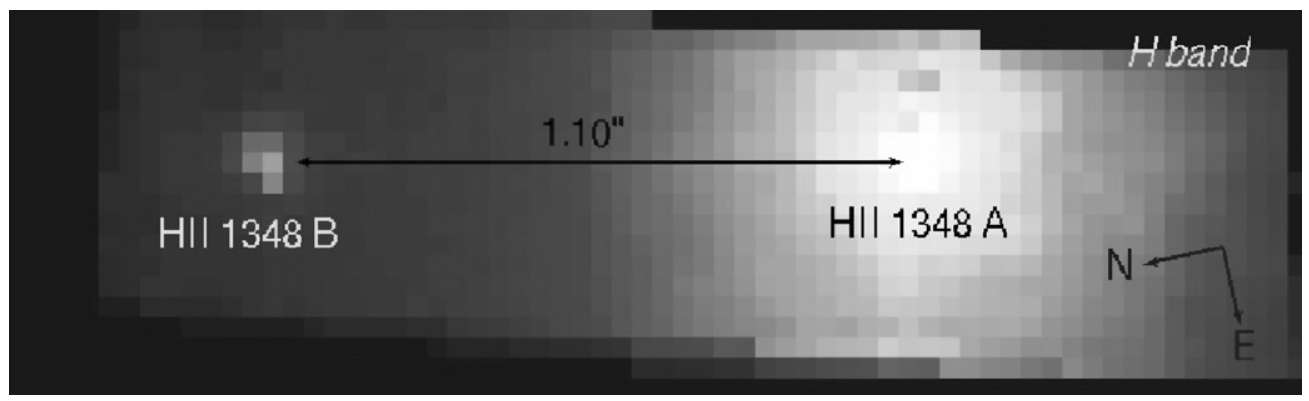

Figure 2. Two-dimensional rendition of the three-dimensional $(x, y, \lambda)$ Keck/OSIRIS $H b b$-band data cube, collapsed along the wavelength direction. The 35 mas pixel size undersamples the FWHM $=35$ mas Keck AO PSF. The total exposure time is 30 minutes, taken in five exposures of 6 minutes.

Table 1

Photometry and Astrometry of H II 1348B

\begin{tabular}{lcccccc}
\hline \hline $\begin{array}{l}\text { Date } \\
(\mathrm{dd} / \mathrm{mm} / \mathrm{yy})\end{array}$ & $\begin{array}{c}\Delta K_{s} \\
(\mathrm{mag})\end{array}$ & $\begin{array}{c}K_{s} \\
(\mathrm{mag})\end{array}$ & $\begin{array}{c}J \\
(\mathrm{mag})\end{array}$ & $\begin{array}{c}H \\
(\mathrm{mag})\end{array}$ & $\begin{array}{c}\Delta \alpha \\
(")\end{array}$ & $\begin{array}{c}\Delta \delta \\
(")\end{array}$ \\
\hline$? ? / 09 / 96^{\mathrm{a}}$ & $5.47[0.01]^{\mathrm{b}}$ & $15.06[0.05]$ & $\ldots$ & $\ldots$ & $-0.23[0.01]$ \\
$03 / 10 / 04$ & $5.15[0.09]$ & $14.88[0.09]$ & $16.04[0.09]$ & $15.30[0.09]$ & $-0.251[0.003]$ \\
$21 / 11 / 05$ & $\cdots$ & $\cdots$ & $\ldots$ & $\cdots$ & $-0.255[0.008]$ \\
\hline
\end{tabular}

Notes.

a The first-epoch data are from Bouvier et al. (1997). The exact date of the observation is not listed, but the data are obtained between 1996 September 25 and 1996 October 1. The R.A. and decl. offsets are calculated from the radial separation (1".09) and position angle (347.9) of the companion, assuming uncertainties of 1\% in pixel scale and 0.3 in orientation. Bouvier et al. (1997) do not list astrometric uncertainties.

b Bouvier et al. (1997) observations were done in $K$ rather than in $K_{s}$ with an apparent $K$-band magnitude of 9.59 mag for H II 1348 A, compared to the Two Micron All Sky Survey $K_{s}$-band magnitude of 9.719 mag used in this paper. Although Bouvier et al. (1997) assign an uncertaintity of 0.01 mag to their photometry, this is inconsistent with normal accuracy of even relative photometry in low-Strehl ratio AO images, and inconsistent with our higher-quality PALAO images, so we believe the error is underestimated. 


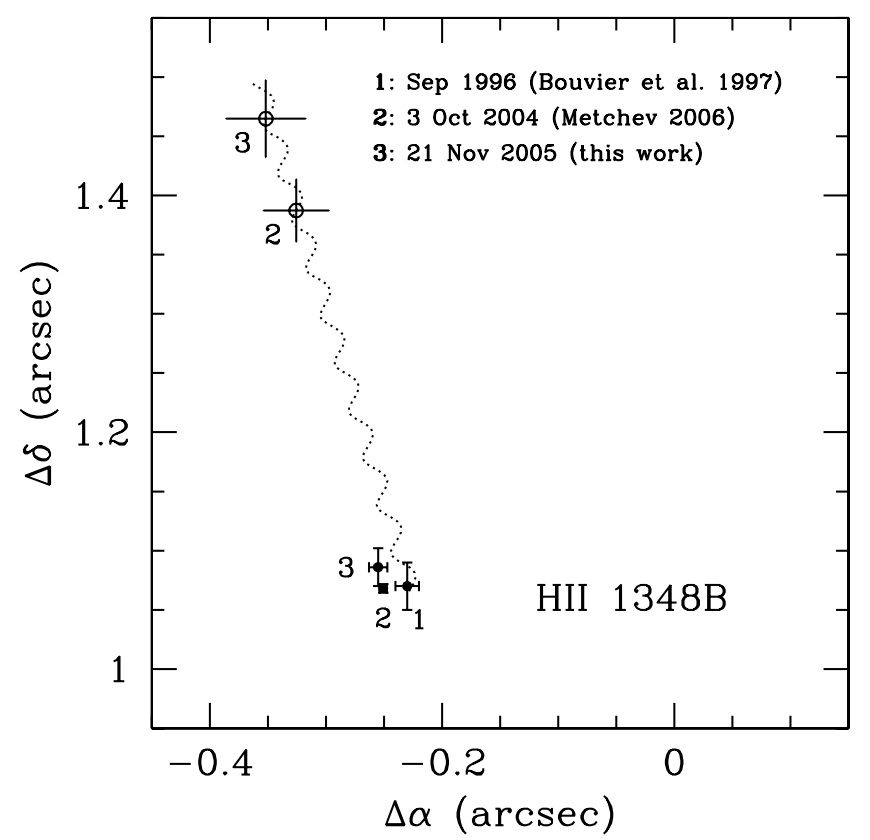

Figure 3. Astrometric measurements of the position of $\mathrm{H}_{\text {II }} 1348 \mathrm{~B}$ relative to its primary star. The solid points represent measurements at three different epochs, spanning 10 years. The dotted curve traces the expected relative motion if the companion were an unrelated background star. The open circles with larger error bars represent the expected location of such a background star at epochs 2 and 3 .

the primary at the location of the tertiary component by locally fitting a quadratic polynomial as a function of both position and wavelength to the radial profile of the halo between $0{ }^{\prime \prime} 12$ and 0 '.56 from H in 1348B.

The wavelength dispersion for the individual extractions was calibrated using the wavelengths of night-sky OH lines. After wavelength calibration, the individual extracted spectra were median-combined, smoothed to the 3.25 pixel $(R \approx 1500)$ resolution of the spectroscopic slit, and then the combined spectrum of H II 1348B was divided by the combined spectrum of the primary to remove telluric features. Given the relatively low dispersion of our observations, the SB2 nature of the primary was not evident in our spectra and did not affect our telluric calibration. The systemic spectral type of the primary is $\mathrm{K} 5$ ( $B-V=1.18 \mathrm{mag}$; Johnson \& Mitchell 1958; Herbig 1962), and so the corrected spectrum of the tertiary component was multiplied by a synthetic K5 V stellar spectrum. The final reduced $K$-band spectrum of $\mathrm{H}_{\mathrm{II}} 1348 \mathrm{~B}$ is shown in Figure 4. The long-slit $K$-band spectrum closely resembles the spectral energy distributions of late M- and early L-type dwarfs. Using a $\chi^{2}$ minimization approach we fit the $K$-band spectrum to spectral standards, limiting the fit to the $2.05-2.30 \mu \mathrm{m}$ wavelength region. The long-slit $K$-band spectrum is best fit by the L1 spectral standard, with an M9 standard giving almost as good of a fit. This marginally disagrees with the integral field unit (IFU) spectra presented next that indicate a slightly earlier, M8 \pm 1 , spectral type. The discrepancy is discussed in Section 3.2.

\subsection{Spectroscopy: Integral Field}

The Keck AO/OSIRIS integral field spectra of H II 1348A/B were obtained with the system aligned along the long side of the $0.56 \times 2$.'.24 FOV. We obtained five $300 \mathrm{~s}$ exposures at $\mathrm{Hbb}$ and four $300 \mathrm{~s}$ exposures at $J b b$, with an extra $300 \mathrm{~s}$ exposure on sky at each band for sky subtraction. An A0 star, HD 24899,

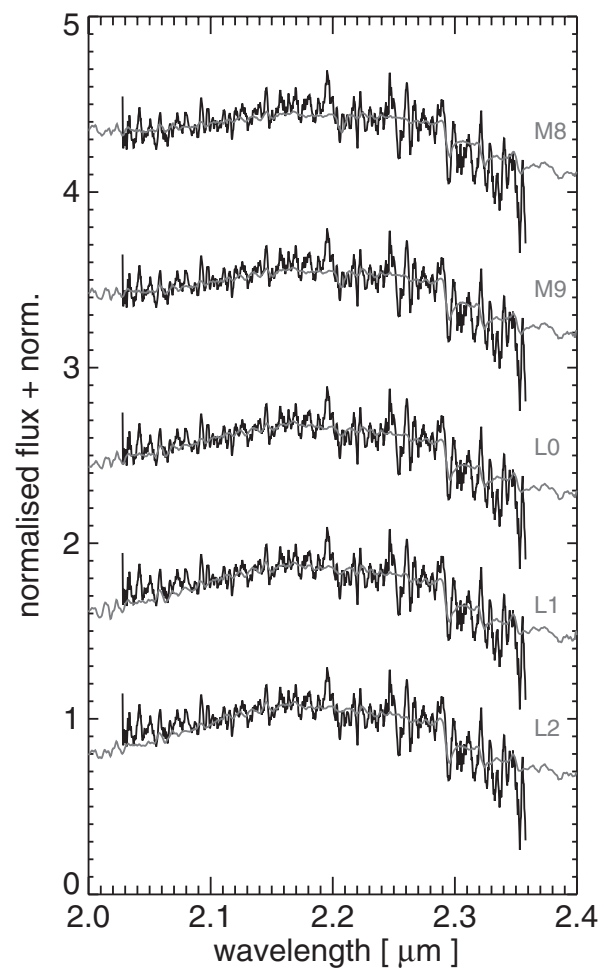

Figure 4. Long-slit $K$-band spectrum of $\mathrm{H}_{\text {II }}$ 1348B smoothed to a resolution of $R \sim 250$. Comparison spectra of GL 644C (M7), GL 752B (M8), LHS 2924 (M9), 2MASSJ0746+2000AB (L0.5), and 2MASSJ0208+2542 (L1) are from the IRTF Spectral Libary (Cushing et al. 2005; Rayner et al. 2009) and have been smoothed to the same resolution.

was observed immediately afterward in each band for telluric calibration.

The data were reduced with the OSIRIS data reduction pipeline (DRP), ${ }^{6}$ following the procedure described in McElwain et al. (2007). We used the default 7 pixel $(0.245)$ radius aperture to extract the spectra of H II 1348A and B, where the center of the aperture was varied with wavelength to account for differential refraction arising both from Earth's atmosphere and from the dichroic in the Keck AO system.

Based on the input set of individual exposures, the OSIRIS DRP produces a single median-combined, wavelengthcalibrated spectrum for each extracted object. A wavelengthcollapsed $H b b$ OSIRIS data cube of H II 1348A/B is shown in Figure 2. The final $J b b$ and $H b b$ spectra of $\mathrm{H}$ II $1348 \mathrm{~B}$ are shown in Figure 5. Comparison spectra of $\mathrm{M}$ and $\mathrm{L}$ dwarfs are from the Infrared Telescope Facility (IRTF) Spectral Libary ${ }^{7}$ (Cushing et al. 2005; Rayner et al. 2009).

To assess the best-fit spectral types of the $J b b$ and $H b b$ spectra, we followed a $\chi^{2}$ minimization when comparing the target spectra and the comparison standards. The $\chi^{2}$ fitting was limited to the $1.185-1.340 \mu \mathrm{m}$ and $1.490-1.800 \mu \mathrm{m}$ wavelength regions, respectively, to avoid regions of high noise. The spectral fitting yields best-fit spectral types of M9 and M7/M8 for the $J b b$ and $H b b$ spectra, respectively.

\section{THE H II 1348 SYSTEM}

\subsection{Probability of Physical Association}

While H II 1348A and H II 1348B likely constitute a physically bound system, there is still a possibility that $\mathrm{H}$ II $1348 \mathrm{~B}$

\footnotetext{
6 http://www2.keck.hawaii.edu/inst/osiris/tools/

7 http://irtfweb.ifa.hawaii.edu/ spex/IRTF_Spectral_Library/
} 

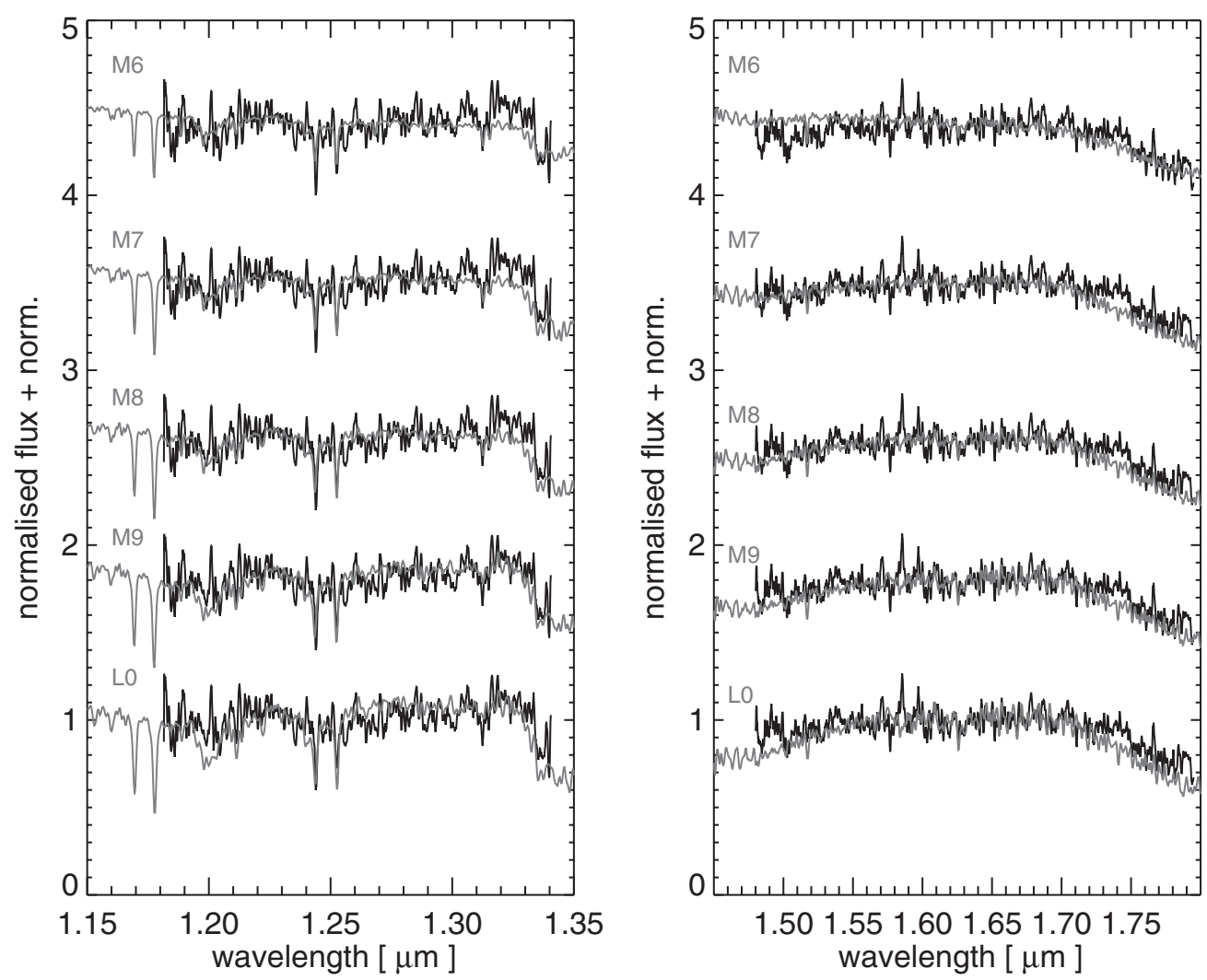

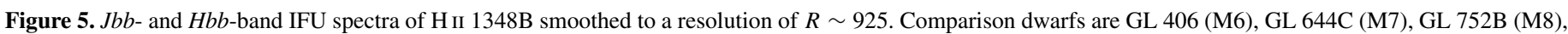

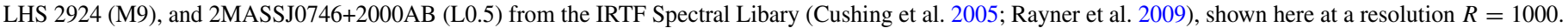

may be an unrelated Pleiades brown dwarf. We estimate the probability for such a spurious alignment by considering the surface density of Pleiades brown dwarfs and the total sky area surveyed by Bouvier et al. (1997) in their AO survey of the Pleiades. (H II 1348B was the only potential substellar companion found by that survey, and we targeted it for confirmation precisely because of its unresolved association status.)

The most comprehensive survey for Pleiades brown dwarfs is that of Moraux et al. (2003), who imaged a total area of $6.4 \mathrm{deg}^{2}$ and discovered 40 brown dwarf candidates as faint as $I=21.7 \mathrm{mag}$, or as low as $\sim 0.03 M_{\odot}$ in mass (Baraffe et al. 1998). The total area surveyed by the Bouvier et al. (1997) AO observations of 144 Pleiades stars is $2.5 \times 10^{-3} \mathrm{deg}^{2}$ : 144 stars $\times$ a $15^{\prime \prime} \times 15^{\prime \prime}$ area imaged around each star. The depth of the Bouvier et al. (1997) survey is $K=17 \mathrm{mag}$, also corresponding to $\mathrm{a} \approx 0.03 M_{\odot}$ lower mass limit. The expected number of $\gtrsim 0.03 M_{\odot}$ brown dwarfs in the Bouvier et al. (1997) AO survey is then $2.5 \times 10^{-3} \mathrm{deg}^{2} / 6.4 \mathrm{deg}^{2} \times 40$ BD's $=0.016$ BD's. The Poisson probability of detecting at least one Pleiades brown dwarf that is not orbiting another Pleiades member is then $P\left(n_{\mathrm{BD}} \geqslant 1\right)=1-P\left(n_{\mathrm{BD}}=0\right)=1-\exp (-0.016)=1.5 \%$.

We therefore conclude that the $\mathrm{H}$ II 1348 spectroscopic binary and its visual tertiary companion form a common proper-motion pair, and that there is a $98 \%$ probability that they form a physically bound multiple system. We will henceforth assume that the visual companion $\mathrm{HII}_{\mathrm{I}} 1348 \mathrm{~B}$ is indeed bound to $\mathrm{H}$ II 1348A.

\subsection{Analysis of the Spectra}

Despite the long-slit $K$-band spectrum pointing toward a later spectral type, we adopt a spectral type of M8 \pm 1 for HII 1348B: the mean of the OSIRIS $J b b$ - and $H b b$-band spectra. We note that the 0 .'13 slit width for the Palomar AO $K$-band long-slit spectrum was only slightly wider than the $0^{\prime \prime} 10 \mathrm{PSF}$, and only $\sim 5$ times wider than the alignment precision for the target on the slit. Rather than oriented along the parallactic angle, the long slit was aligned along the visual binary components. Both factors may lead to wavelengthdependent slit looses, either through inadequate centering of the target on the slit, or through differential atmospheric refraction. These incur continuum gradients that systematically affect the inferred spectral type of the companion, especially in cases where the spectral type is based on the continuum slope or on broadband molecular absorption features at either end of the spectrum (see discussion in Goto et al. 2003; McElwain et al. 2007). A variation in continuum slope could also be caused by an increased error in the telluric correction. The thicker atmospheric layer at Palomar Observatory causes greater uncertainty in the telluric correction of the $K$-band data, compared to the OSIRIS $J b b$ - and $H b b$-band taken at Mauna Kea, and therefore more uncertainty in the spectral type, which is partly based on $\mathrm{H}_{2} \mathrm{O}$ depression.

The OSIRIS $J b b$-band spectrum covers the gravity sensitive K I doublet at $1.243 / 1.252 \mu \mathrm{m}$. In young dwarfs, low gravity causes the alkali lines to appear weaker and sharper than in normal (older) dwarfs (Steele et al. 1995; Martin et al. 1996; Kirkpatrick et al. 2008). Thus, by comparing the shape and strengths of the K I doublet to a normal M8 dwarf, we should be able to infer if $\mathrm{H}$ II $1348 \mathrm{~B}$ has lower gravity than the comparison dwarf. Figure 6 shows such a comparison. The $\mathrm{K}_{\mathrm{I}}$ line at $1.243 \mu \mathrm{m}$ is slightly redshifted and much stronger than expected. The offset in position remains unexplained at present, but may have to do with varying contamination from the much brighter primary component because of cross-talk in the data from the 


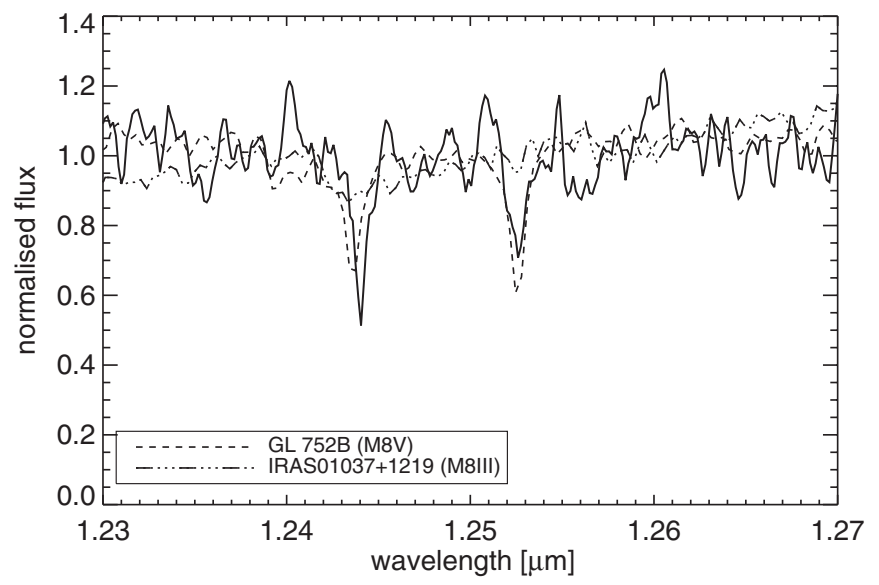

Figure 6. Zoom of the $\mathrm{K}_{\mathrm{I}}$ doublet of $\mathrm{H}_{\mathrm{II}}$ 1348B showing an apparent redshift in the $1.243 \mu \mathrm{m}$ line of the doublet, possibly due to a detector cross-talk effect.

early commissioning days of OSIRIS. Disregarding the $\mathrm{K}_{\mathrm{I}}$ line at $1.243 \mu \mathrm{m}$, the $\mathrm{K} \mathrm{I}$ line at $1.252 \mu \mathrm{m}$ appears to be intermediate in strength between the giant and the dwarf comparison spectra. However, given the fact that the $\mathrm{K}_{\mathrm{I}}$ line strength is consistent within the noise of our data, we can draw no reliable conclusions on the gravity of H II 1348B.

\subsection{The Mass of H II $1348 B$}

Given the uncertainty in the absolute distance to the Pleiades, with measurements varying between $120 \mathrm{pc}$ and $140 \mathrm{pc}$, we consider two distances, $120.2 \pm 1.9 \mathrm{pc}$ (based on the revised Hipparcos measurements by van Leeuwen 2009) and 133 pc (a weighted mean of trigonometric and orbital parallax distances from Pan et al. 2004, Munari et al. 2004, Zwahlen et al. 2004, and Southworth et al. 2005) when calculating the absolute magnitudes and luminosity of $\mathrm{H}$ II 1348B (Table 2). Estimates for the age of the Pleiades range between $100 \mathrm{Myr}$ and $125 \mathrm{Myr}$ (Meynet et al. 1993; Stauffer et al. 1998).

The models of Baraffe et al. (1998) do not include absolute magnitudes as faint as those of $\mathrm{H}$ II $1348 \mathrm{~B}$ at an age of $0.1 \mathrm{Gyr}$, implying that the objects mass is below the hydrogen burning limit. Averaging the mass estimates (Chabrier et al. 2000) given by the absolute $J, H$, and $K_{s}$ magnitudes reported in this paper, yields masses of $0.056 \pm 0.003$ and $0.063 \pm 0.004$ at $120 \mathrm{pc}$ and $133 \mathrm{pc}$, respectively. The corresponding luminosity estimates from the Lyon models are listed in Table 2.

Likewise, we can estimate the mass of H II 1348B via its luminosity. Using measured bolometric corrections for M8-type dwarfs (Dahn et al. 2002; Golimowski et al. 2004; Leggett et al. 2002), we calculated the bolometric magnitude and luminosity of H II 1348B (Table 2). At an age of 100-125 Myr and a distance of $120 \mathrm{pc}$, models from Burrows et al. (1997) and Chabrier et al. (2000) yield mass estimates between 0.053 and $0.055 M_{\odot}$ (Figure 7).

The mass of $\mathrm{H}$ II $1348 \mathrm{~B}$ is within the range of mass estimates for other Pleiads with similar spectral types. Bouvier et al. (1998) give a mass of $0.061 M_{\odot}$ (at a distance of $125 \mathrm{pc}$ ) for the M6 dwarf CFHT PL 14 (Stauffer et al. 1998). PPl 1 is a M6.5 \pm 0.5 dwarf with an estimated mass of $\sim 0.074 M_{\odot}$ (Bihain et al. 2010) and is right at the lithium depletion edge in the Pleaides (Stauffer et al. 1998). Calar 3 and Teide 1, two M8 dwarfs, have estimated masses of $\sim 0.054 M_{\odot}$ and $\sim 0.052 M_{\odot}$ (at $d=120 \mathrm{pc}$; Bihain et al. 2010), respectively, and their substellar nature is confirmed through the presence of lithium
Table 2

Parameters of H II 1348B

\begin{tabular}{lcc}
\hline \hline & $120 \mathrm{pc}$ & $133 \mathrm{pc}$ \\
\hline$m-M$ & $5.40[0.03]$ & $5.62[0.03]$ \\
$M_{J}(\mathrm{mag})$ & $10.64[0.10]$ & $10.42[0.10]$ \\
$M_{H}(\mathrm{mag})$ & $9.90[0.10]$ & $9.68[0.10]$ \\
$M_{K}(\mathrm{mag})$ & $9.48[0.10]$ & $9.26[0.10]$ \\
$a(\mathrm{AU})$ & $131.0[2.2]$ & $145.0[2.3]$ \\
\hline & Via absolute magnitudes & \\
\hline $\log L / L_{\odot}$ & $-3.16[0.05]$ & $-3.05[0.06]$ \\
$M_{B}\left(M_{\odot}\right)$ & $0.056[0.003]$ & $0.063[0.004]$ \\
$q$ & $0.046[0.004]$ & $0.052[0.005]$ \\
$\mathrm{EB}\left(10^{41} \mathrm{erg}\right)$ & $92.0[8.5]$ & $93.5[9.2]$ \\
\hline & Via bolometric corrections & \\
\hline $\log L / L_{\odot}$ & $-3.14[0.08]$ & $-3.06[0.08]$ \\
\hline & Chabrier et al. (2000) & $0.061[0.008]$ \\
\hline$M_{B}\left(M_{\odot}\right)$ & $0.055[0.007]$ & $0.050[0.008]$ \\
$q$ & $0.045[0.007]$ & $90.6[13.7]$ \\
$\mathrm{EB}\left(10^{41} \mathrm{erg}\right)$ & $90.4[13.4]$ & $0.047[0.007]$ \\
\hline & Burrows et al. (1997) & \\
\hline$M_{B}\left(M_{\odot}\right)$ & $0.053[0.007]$ & \\
$q$ & $0.043[0.007]$ & {$[13.5]$} \\
$\mathrm{EB}\left(10^{41} \mathrm{erg}\right)$ & $87.1[13.3]$ & \\
\hline & & \\
\hline & & \\
\hline & &
\end{tabular}

(Rebolo et al. 1996). H II 1348B has the same spectral type as Calar 3 and Teide 1, and we therefore conclude that it is also substellar. Future optical spectroscopy could confirm the presence of lithium in $\mathrm{H}$ II 1348B.

\subsection{Mass Ratio}

H II 1348A is a double-lined spectroscopic binary (Queloz et al. 1998), and hence an upper mass limit for the primary can be obtained by assuming that both components are K5 V stars. With the mass of a single K5 V star being $\sim 0.65 M_{\odot}$ (Zakhozhaj 1998), the SB2 star H II 1348Aab has a mass of $\sim 1.3 M_{\odot}$. A more precise mass estimate can be obtained using the $B-V$ colors of $\mathrm{H}$ II $1348 \mathrm{Aa}$ and $\mathrm{H}$ II $1348 \mathrm{Ab}$ (1.05 and 1.35 , respectively) given by Queloz et al. (1998). The colors roughly translate to masses of $0.67 \pm 0.07 M_{\odot}$ and $0.55 \pm$ $0.05 M_{\odot}$, respectively, yielding a total estimated mass of $1.22 \pm$ $0.09 M_{\odot}$ for H II 1348Aab. Adopting the latter mass for the SB2 component, the mass ratio of $\mathrm{H}$ II $1347 \mathrm{~B}$ to $\mathrm{H}$ II $1347 \mathrm{Aab}$ is between 0.043 and 0.052 (Table 2). This is the lowest among the known Pleiad multiples (Bouvier et al. 1997; Bouy et al. 2006) and is comparable to that of very low mass ratio binaries in the field (Faherty et al. 2011).

\section{DISCUSSION AND CONCLUSION}

H II 1348B is a new M8 brown dwarf member of the Pleiades and the first substellar companion discovered around a Pleiades star. Given that no other substellar companions were discovered in the Bouvier et al. (1997) survey at similar or wider separations, it is worth considering whether H II 1348B may be unusually weakly bound, compared to other binary systems in the Pleiades or in the field.

Bouvier et al. (1997) found a total of 28 stellar binaries in the Pleiades in their CHFT AO survey. Hubble Space Telescope surveys of very low mass stars and brown dwarfs conducted by Martín et al. (2003) and Bouy et al. (2006) revealed three additional binaries. In Figure 8 we compare the binding energies 

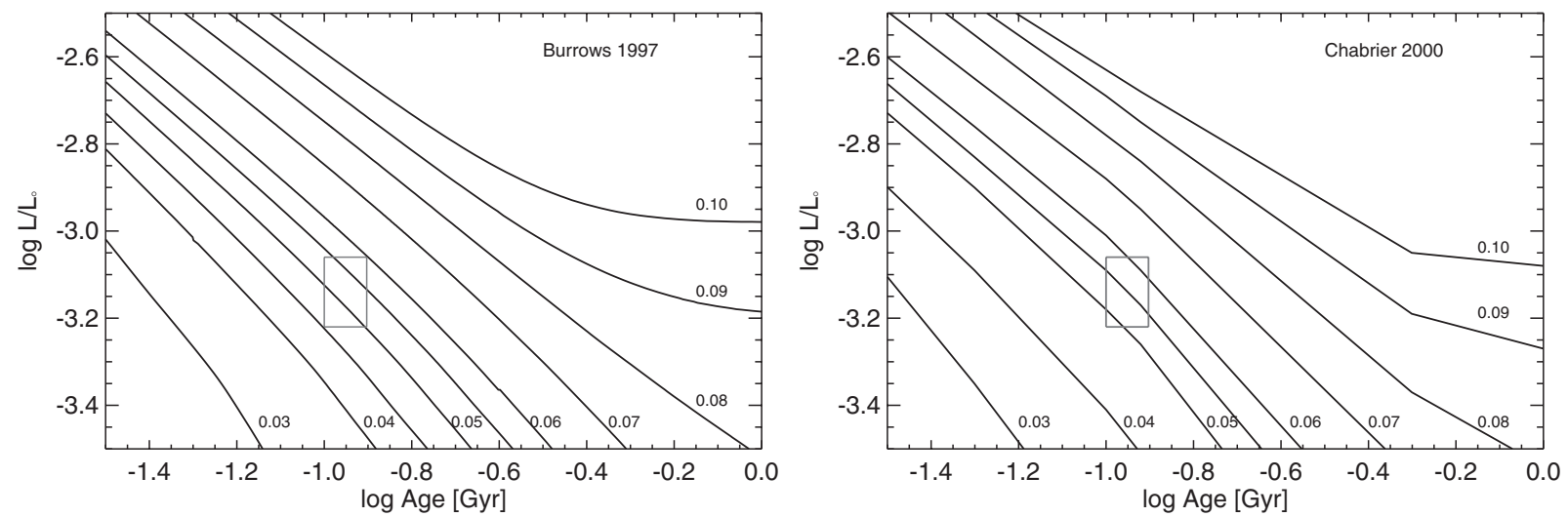

Figure 7. Plot of the Burrows et al. (1997) and Chabrier et al. (2000) evolutionary models. The box indicates the allowed range of parameters (age and $L_{\text {bol }}$ ) for H II 1348 B at a distance of $120 \mathrm{pc}$.

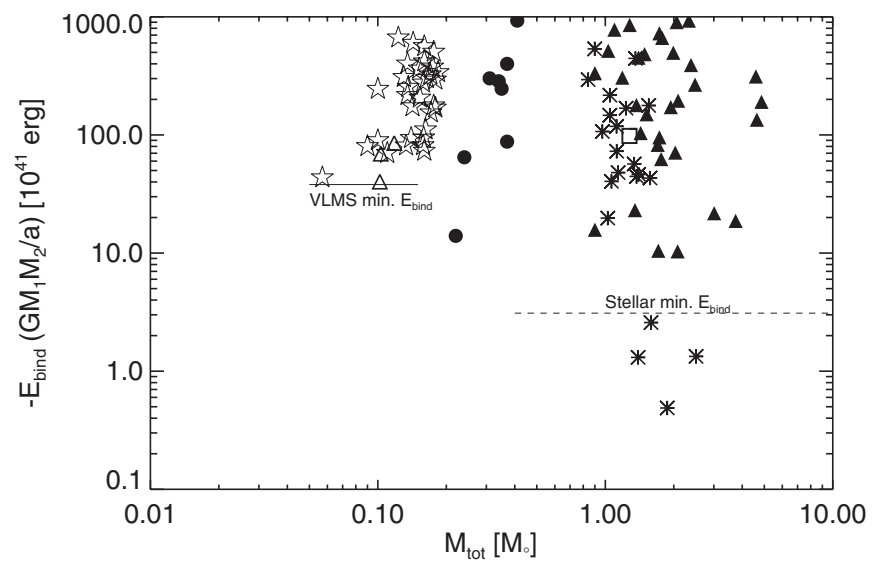

Figure 8. Comparison of the binding energy of stellar and very low mass (VLM) binary and multiple systems (Close et al. 2003, 2007). The symbols are: filled triangles - stellar binaries and multiples (Close et al. 1990), filled circles-lowmass Hyades binaries (Reid \& Gizis 1997), open stars-VLM binares (Close et al. 2003, and references therein), asterisks_-stellar Pleiades binaries (Bouvier et al. 1997), open triangles - VLM Pleiades binaries (Bouy et al. 2006), and open square-H II 1348.

of these systems, and those of field A-M binaries (Close et al. $1990,2003,2007)$ to the binding energy of H II $1348 \mathrm{~A} / \mathrm{B}$. As can be seen, H II 1348A/B sits in the middle of the locus for stellar binaries and is comparably or more tightly bound even than the three very low mass Pleiades binaries. What is more, substellar companions up to 10 times further away from their primaries would still be well above the minimum stellar binding energy in the Pleiades.

The dearth of known brown dwarf companions to stars in the Pleiades may thus be attributable to the lack of a followup sensitive and comprehensive high-contrast imaging survey of the cluster. Small samples of Pleiades stars have since been observed in deep surveys by Metchev \& Hillenbrand (2009; 23 stars) and Tanner et al. (2007; 14 stars), with no new brown dwarf companion detections. However, a much more comprehensive survey is needed to reveal brown dwarf companions with any statistical confidence (Metchev \& Hillenbrand 2009).

Current AO systems at Keck or Gemini North should allow the detection of companions with masses down to $\sim 0.03 M_{\odot}$ at separations larger than $\sim 60 \mathrm{AU}$. Using the Gemini North AO system Altair in combination with NIRI, Lafrenière et al. (2007) showed that companions up to 9.5 mag fainter can be detected at separations of $>0$ '.5 from the primary. Accounting for the narrowband filter used during the observation, brown dwarfs down to $0.03 M_{\odot}$ should be detectable around bright ( $V$ $<12$ mag) Pleiades stars. Likewise, using angular differential imaging (Marois et al. 2006) in combination with the Keck AO system, Metchev et al. (2009) demonstrated that companions up to $\Delta H=10.5$ mag fainter than the primary can be detected at separations larger than 0.5 . Thus, at the distance of the Pleiades brown dwarfs down to $0.02 M_{\odot}$ should be detectable at separations larger than $\sim 60 \mathrm{AU}$. Upcoming instruments like the Gemini Planet Imager (Macintosh et al. 2008; McBride et al. 2011) and the extreme AO system at Palomar (PALM3000; Bouchez et al. 2008) will enable detections of brown dwarf companions at even smaller separation down to 0.2 .

Given the importance of the Pleiades as an age and a dynamical benchmark for stellar and substellar evolution, a deep AO survey could have a high impact in delivering substellar objects much fainter and cooler than the ones presently known from wide-area surveys. While wide-area surveys are more efficient in discovering large numbers of substellar candidate members, the limited precision of seeing-limited astrometry, and the increasingly challenging radial velocity measurements at fainter magnitudes, prevents the unequivocal confirmation of the lower mass candidates as bona fide members. The factor of $\sim 100$ higher precision attainable over narrow angles with $\mathrm{AO}$ leaves little doubt about the astrometric association of candidate binaries and is thus also an excellent tool for confirming the cluster membership of even the lowest-mass, faintest companions.

Partial support for S.A.M. was provided through the Spitzer Fellowship Program under award 1273192. The authors also wish to extend special thanks to those of Hawaiian ancestry on whose sacred mountain of Mauna Kea we are privileged to be guests.

\section{Facilities: Keck:II, Hale}

Note added in proof. Shortly after the manuscript was accepted for publication, another brown dwarf companion in the Pleiades, HD 23514B, was announced by Rodriguez et al. (2011).

\section{REFERENCES}

Baraffe, I., Chabrier, G., Allard, F., \& Hauschildt, P. H. 1998, A\&A, 337, 403 Bihain, G., Rebolo, R., Zapatero Osorio, M. R., Béjar, V. J. S., \& Caballero, J. A. 2010, A\&A, 519, A93

Bouchez, A. H., Dekany, R. G., Angione, J. R., et al. 2008, Proc. SPIE, 7015, $70150 Z$

Bouvier, J., Rigaut, F., \& Nadeau, D. 1997, A\&A, 323, 139 
Bouvier, J., Stauffer, J. R., Martin, E. L., et al. 1998, A\&A, 336, 490 Bouy, H., Moraux, E., Bouvier, J., et al. 2006, ApJ, 637, 1056 Burrows, A., Marley, M., Hubbard, W. B., et al. 1997, ApJ, 491, 856

Chabrier, G., Baraffe, I., Allard, F., \& Hauschildt, P. 2000, ApJ, 542, 464

Close, L. M., Richer, H. B., \& Crabtree, D. R. 1990, AJ, 100, 1968

Close, L. M., Siegler, N., Freed, M., \& Biller, B. 2003, ApJ, 587, 407

Close, L. M., Zuckerman, B., Song, I., et al. 2007, ApJ, 660, 1492

Cushing, M. C., Rayner, J. T., \& Vacca, W. D. 2005, ApJ, 623, 1115

Dahn, C. C., Harris, H. C., Vrba, F. J., et al. 2002, AJ, 124, 1170

Dekany, R. G., Brack, G., Palmer, D., et al. 1998, Proc. SPIE, 3353, 56

Faherty, J. K., Burgasser, A. J., Bochanski, J. J., et al. 2011, AJ, 141, 71

Golimowski, D. A., Leggett, S. K., Marley, M. S., et al. 2004, AJ, 127, 3516

Goto, M., Hayano, Y., Kobayashi, N., et al. 2003, Proc. SPIE, 4839, 1117

Hartkopf, W. I., \& Mason, B. D. 2011, http://ad.usno.navy.mil/wds/orb6.html

Hayward, T. L., Brandl, B., Pirger, B., et al. 2001, PASP, 113, 105

Herbig, G. H. 1962, ApJ, 135, 736

Johnson, H. L., \& Mitchell, R. I. 1958, ApJ, 128, 31

Kirkpatrick, J. D., Cruz, K. L., Barman, T. S., et al. 2008, ApJ, 689, 1295

Lafrenière, D., Doyon, R., Marois, C., et al. 2007, ApJ, 670, 1367

Larkin, J., Barczys, M., Krabbe, A., et al. 2006, New Astron. Rev., 50, 362

Leggett, S. K., Golimowski, D. A., Fan, X., et al. 2002, ApJ, 564, 452

Macintosh, B. A., Graham, J. R., Palmer, D. W., et al. 2008, Proc. SPIE, 7015, 701518

Marois, C., Lafrenière, D., Doyon, R., Macintosh, B., \& Nadeau, D. 2006, ApJ, 641, 556

Martín, E. L., Barrado y Navascués, D., Baraffe, I., Bouy, H., \& Dahm, S. 2003, ApJ, 594, 525

Martín, E. L., Brandner, W., Bouvier, J., et al. 2000, ApJ, 543, 299

Martin, E. L., Rebolo, R., \& Zapatero-Osorio, M. R. 1996, ApJ, 469, 706

McBride, J., Graham, J. R., Macintosh, B., et al. 2011, PASP, 123, 692
McElwain, M. W., Metchev, S. A., Larkin, J. E., et al. 2007, ApJ, 656, 505 Mermilliod, J.-C., Rosvick, J. M., Duquennoy, A., \& Mayor, M. 1992, A\&A, 265,513

Metchev, S., Marois, C., \& Zuckerman, B. 2009, ApJ, 705, L204

Metchev, S. A. 2006, PhD thesis, California Institute of Technology, California

Metchev, S. A., \& Hillenbrand, L. A. 2004, ApJ, 617, 1330

Metchev, S. A., \& Hillenbrand, L. A. 2009, ApJS, 181, 62

Meynet, G., Mermilliod, J.-C., \& Maeder, A. 1993, A\&AS, 98, 477

Moraux, E., Bouvier, J., Stauffer, J. R., \& Cuillandre, J.-C. 2003, A\&A, 400, 891

Munari, U., Dallaporta, S., Siviero, A., et al. 2004, A\&A, 418, L31

Pan, X., Shao, M., \& Kulkarni, S. R. 2004, Nature, 427, 326

Queloz, D., Allain, S., Mermilliod, J.-C., Bouvier, J., \& Mayor, M. 1998, A\&A, 335,183

Rayner, J. T., Cushing, M. C., \& Vacca, W. D. 2009, ApJS, 185, 289

Rebolo, R., Martin, E. L., Basri, G., Marcy, G. W., \& Zapatero-Osorio, M. R. 1996, ApJ, 469, L53

Reid, I. N., \& Gizis, J. E. 1997, AJ, 114, 1992

Rodriguez, D. R., Marois, C., Zuckerman, B., Macintosh, B., \& Melis, C. 2011, ApJ (arXiv:1112.4815)

Southworth, J., Maxted, P. F. L., \& Smalley, B. 2005, A\&A, 429, 645

Stauffer, J. R. 1984, ApJ, 280, 695

Stauffer, J. R., Schultz, G., \& Kirkpatrick, J. D. 1998, ApJ, 499, L199

Steele, I. A., Jameson, R. F., Hodgkin, S. T., \& Hambly, N. C. 1995, MNRAS, 275,841

Tanner, A., Beichman, C., Akeson, R., et al. 2007, PASP, 119, 747

Troy, M., Dekany, R. G., Brack, G., et al. 2000, Proc. SPIE, 4007, 31 van Leeuwen, F. 2009, A\&A, 497, 209

Wizinowich, P., Acton, D. S., Shelton, C., et al. 2000, PASP, 112, 315

Zakhozhaj, V. A. 1998, VizieR Online Data Catalog, 5101, 0

Zwahlen, N., North, P., Debernardi, Y., et al. 2004, A\&A, 425, L45 